\title{
Topical Application of Retinyl Palmitate-Loaded Nanotechnology-Based Drug Delivery Systems for the Treatment of Skin Aging
}

\author{
Marcela B. Oliveira, ${ }^{1}$ Alice Haddad do Prado, ${ }^{1}$ Jéssica Bernegossi, ${ }^{1}$ \\ Claudia S. Sato, ${ }^{1}$ Iguatemy Lourenço Brunetti, ${ }^{2}$ Maria Virgínia Scarpa, \\ Gislaine Ricci Leonardi, ${ }^{3}$ Stig E. Friberg, ${ }^{4}$ and Marlus Chorilli ${ }^{1}$ \\ ${ }^{1}$ Departament of Drugs and Pharmaceuticals, School of Pharmaceutical Sciences, UNESP, 14801-902 Araraquara, SP, Brazil \\ ${ }^{2}$ Departament of Clinical Analysis, School of Pharmaceutical Sciences, UNESP, 14801-902 Araraquara, SP, Brazil, Brazil \\ ${ }^{3}$ Institute of Environmental, Chemistry and Pharmaceuticals Sciences, Federal University of São Paulo (UNIFESP), \\ 09972-270 Diadema, SP, Brazil \\ ${ }^{4}$ Ugelstad Laboratory, Norwegian University of Science and Technology (NTNU), 7491 Trondheim, Norway
}

Correspondence should be addressed to Marlus Chorilli; chorilli@fcfar.unesp.br

Received 12 November 2013; Revised 9 January 2014; Accepted 31 January 2014; Published 19 March 2014

Academic Editor: Philippe Humbert

Copyright @ 2014 Marcela B. Oliveira et al. This is an open access article distributed under the Creative Commons Attribution License, which permits unrestricted use, distribution, and reproduction in any medium, provided the original work is properly cited.

The objective of this study was to perform a structural characterization and evaluate the in vitro safety profile and in vitro antioxidant activity of liquid crystalline systems (LCS) with and without retinyl palmitate (RP). LCS containing polyether functional siloxane (PFS) as a surfactant, silicon glycol copolymer (SGC) as oil phase, and water in the ratios 30:25:45 and 40:50:10 with (OLS, $=$ RP-loaded opaque liquid system and TLS $_{v}=$ RP-loaded transparent liquid system, respectively) and without (OLS and TLS, respectively) RP were studied. Samples were characterized using polarized light microscopy (PLM) and rheology analysis. In vitro safety profile was evaluated using red cell hemolysis and in vitro cytotoxicity assays. In vitro antioxidant activity was performed by the DPPH method. PLM analysis showed the presence of lamellar LCS just to TLS. Regardless of the presence of RP, the rheological studies showed the pseudoplastic behavior of the formulations. The results showed that the incorporation of RP in LCS improved the safety profile of the drug.In vitro antioxidant activity suggests that LCS presented a higher capacity to maintain the antioxidant activity of RP. PFS-based systems may be a promising platform for RP topical application for the treatment of skin aging.

\section{Introduction}

Currently, the demand for products that reduce skin aging is constantly growing because people want to stay young as long as possible. Cosmeceuticals (cosmetics producing beneficial results for the body, for example, the effects of antifree radicals) represent one of the largest growing segments of the skin care market, especially for products that are designed to prevent and treat skin aging $[1,2]$.

Aging is a natural and inevitable process that reverses the biological characteristics acquired during development and leads to cell death [3]. The natural process of skin rejuvenation slows dramatically and the skin becomes thinner and drier, losing elasticity [4]. Skin aging is influenced by several factors including genetic factors, environmental exposures (ultraviolet radiation (UV), xenobiotics, and stress mechanisms), hormonal changes, and metabolic processes (generation of reactive oxygen species as chemically activated sugars and aldehydes). All of these factors act together in changing the structure, function, and appearance of the skin [5].

The use of retinoids has been highlighted for the treatment of photoaging, and there are many different brands and formulations currently available in the market that employ such substances. A randomized clinical study showed beneficial results of retinoids in photoaging, in addition to repairing and preventing skin aging [6]. 


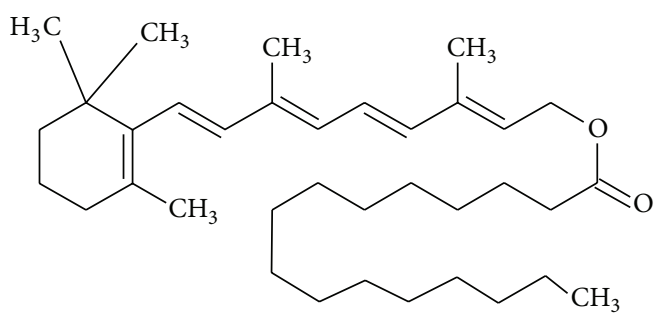

FIGURE 1: Molecular structure of retinyl palmitate.

Studies have indicated that retinoids may have specific effects on the receptor resulting in decreased skin roughness and skin facial wrinkles [7]. A study with 24 Korean women for 24 weeks showed improvement in skin roughness and fine wrinkles $[8,9]$.

Retinyl palmitate, whose molecular structure is shown in Figure 1, is an ester of retinol and is the major form of vitamin A found in the epidermis. This compound has been widely used in pharmaceutical and cosmetic formulations [3]. It has a high molecular weight and a stable formulation. To be active, RP should be enzymatically converted in the skin to retinol by cleavage of the ester linkage and must then be converted to tretinoin via oxidative processes. The topical administration of RP for 14 days in rats resulted in increased protein and collagen and an epidermal thickening [6].

The main obstacle to the use of topical retinoids is the high incidence of skin irritation. Patients may develop dermatitis with redness and tenderness of the skin. This usually occurs within two to four weeks after initiation of treatment and usually disappears when the treatment is continued. Nevertheless, many patients discontinue therapy because of these reactions. It has been found that derivatives of retinol such as RP do not produce the same irritant effects as retinoic acid and induce the same cellular and molecular changes observed with the application of retinoic acid [10].

Sorg et al. (2005) demonstrated that, although the amounts of RP naturally present in the epidermis are too low to provide effective and efficient protection against UV radiation, these retinoids can easily be administered topically in order to promote effective protection. This was confirmed by some studies, including one conducted in human volunteers who were subjected to UVB light to assess DNA damage and erythema. The participants were treated with a commercial sunscreen (octyl methoxycinnamate) and one containing RP, and it was observed that RP inhibited the formation of thymine dimers (an indicator of DNA damage) and erythema [3]. Another study showed that DNA damage and apoptosis were inhibited in mice by the application of topical retinoic acid, retinaldehyde, retinol, and RP [11].

Currently, the development of pharmaceutical and cosmetic technology is not restricted only to the discovery of new molecules but also to the development of new systems to deliver active ingredients or optimize their release [12]. The combination of consumer desire and the development of these technologies has led to the emergence of new systems with specific properties, capable of incorporating substances with different profile releases optimized for application on different areas [13]. Among the systems developed or with applicability for topical skincare cosmetics, nanotechnologybased drug delivery systems are promising vehicles for dermal and transdermal release of active compounds, particularly the liquid crystalline systems (LCS), multiple emulsions, and nanoemulsions $[13,14]$. LCS have applications in cosmetic and pharmaceutical formulations. They are able to promote the encapsulation of active ingredients, allowing for sustained release of the same, as well as giving protection of drugs and photosensitivity depending on the microstructure, increasing the stability of these systems to reduce coalescence and change in viscosity $[13,15,16]$.

Furthermore, LCS can maintain therapeutic response for a longer period of time, improve drug efficacy and solubility, decrease side effects, and interfere in skin hydration. The incorporation of RP within LCS phases provided a significant reduction in the orbicular wrinkles of human volunteers [17].

Studies involving nanotechnology seek to implement new technological approaches to extend the benefits provided by drug delivery systems. The development of an effective, safe, and reliable carrier system which exhibits good bioavailability and pharmacodynamics and also reduces side effects is a goal for many researchers. Therefore, finding a system that meets those requirements would be extremely valuable and SLC are potential candidates $[13,18]$.

The objective of the present study was to perform a structural characterization and evaluate the in vitro safety profile and antioxidant activity of lamellar LCS, with and without RP.

\section{Materials and Methods}

2.1. Materials. Polyether functional siloxane, DC 5329 (S), and silicon glycol copolymer, DC 193 (O), were purchased from Dow Corning (Michigan, USA), and retinyl palmitate (RP) 1,000,000 IU/g was purchased from Roche (GreenzachWyhlen, Germany). High purity water (W) from a Millipore Milli-Q plus purification system was used throughout.

2.2. Formulation Preparation. The preparation has been described previously [17]. The samples were prepared by heating a mixture of $\mathrm{O}$ and $\mathrm{S}$ to $45^{\circ} \mathrm{C}$. W was heated to $40^{\circ} \mathrm{C}$ and then carefully added under gentle and constant stirring until the mixture reached room temperature. The resulting systems, containing various proportions of the components, were characterized using a pseudoternary phase diagram in order to define the proportions that form LCS [17]. The proportions of each component were calculated from titrations of the binary mixtures of oil phase and surfactant with water. The transitions from an opaque semisolid phase to a transparent viscous system (TVS), viscous and opaque system (OVS), and an opaque liquid system (OLS), as well as a transparent liquid system (TLS) and phase separation (PS), were defined. Diagrams were produced for the RP-loaded (1\%) and RP-unloaded mixtures.

2.3. Polarized Light Microscopy. A small amount of the formulations was placed on a glass slides, covered with 
a cover slip, and examined with the aid of a polarized light microscope (Jenamed 2, Carl Zeiss-Jena) to judge the homogeneity of the dispersions and the presence of optically anisotropic areas by the patterns formed with the sample between crossed polarizers.

\subsection{Rheology Analysis. The rheological determination of for-} mulations was carried out with a controlled-stress rheometer (model RS-1, Haake RheoStress) with plate-plate geometry. This geometry consists of two stainless steel plates $2 \mathrm{~cm}$ in diameter with a gap of $200 \mu \mathrm{m}$ between the plates. Samples were carefully applied to the lower plate, ensuring that formulation shearing was minimized, and allowed to equilibrate for at least 3 minutes prior to analysis. The experiments were carried out with shear rates in the range of $0.001-30 \mathrm{~s}^{-1}$. The shear rate region used was selected on the basis of the strength of resistance to the applied stresses. The rheological measurements were performed on both the up and down curve. All rheological determinations were carried out on all samples at $25.0 \pm 0.2^{\circ} \mathrm{C}$.

\subsection{In Vitro Biological Assays}

2.5.1. Erythrocyte Hemolysis. The erythrocyte hemolysis assay was performed using the experimental procedure described by Jumaa et al. [19] and Huang et al. [20]. Briefly, before use, freshly collected human blood (O positive) was washed three times with $0.01 \mathrm{M}$ Tris- $\mathrm{HCl}$ with a $\mathrm{pH} 7.4$ containing $0.15 \mathrm{M} \mathrm{NaCl}$ (Tri-saline). A suspension of $1 \%(\mathrm{v} / \mathrm{v})$ erythrocytes was prepared with packed red blood cells resuspended in Tris-saline. RP-loaded LCS, RP-unloaded LCS, and $\mathrm{RP}$ free were dissolved in Tris-saline to a final concentration of $27 \mu \mathrm{M}$. As a positive control (100\% lysis), a $1 \%(\mathrm{v} / \mathrm{v})$ Triton X-100 solution was used. After incubation for 1 hour at $37^{\circ} \mathrm{C}$, the samples were centrifuged at $3000 \times \mathrm{g}$ for 2 minutes. Aliquots of $100 \mu \mathrm{L}$ of the supernatant were transferred to 96-well microplates, and the absorbance was determined at $405 \mathrm{~nm}$ using a BioRad Model 3550-UV (USA) microplate reader. The assay was performed in triplicate. The percentage of hemolysis was calculated using the following equation: $\%$ hemolysis $=$ (absorbance of the test sample/absorbance at $100 \%$ lysis $) \times 100$.

2.5.2. In Vitro Nonspecific Cytotoxicity. In vitro testing for analysis of the cytotoxicity of the formulations was performed using J-774 mouse macrophages as template. Cells were seeded in bottom microplates (Nunclon) with 96 wells and a density of $2.5-10.0 \times 10^{5}$ cells/well with different doses of the formulation and free $\mathrm{RP}(18.6,10,5$, and $1 \mu \mathrm{M})$ or vehicle control for 48 hours. The cells were washed with PBS after removal of the compounds and cell viability was assessed by colorimetry formazan (MTT).

The method of 3 [4,5-dimethylthiazol-2-yl]-2,5-diphenyltetrazolium bromide (MTT) is simple, reliable, and reproducible colorimetric method for measuring mitochondrial metabolic reduction of yellow tetrazolium salt to the insoluble formazan crystals in aqueous solution of viable cells. Cells and MTT $(0.4 \mathrm{mg} / \mathrm{mL})$ were incubated at $37^{\circ} \mathrm{C}$ for 3 hours.
Subsequently, the supernatant was removed and formazan crystals were dissolved in DMSO $(180 \mathrm{~mL})$. The plates were agitated for 10 minutes and optical density was measured using a multiwall spectrophotometer operating at $560 \mathrm{~nm}$. Concentrations were tested in triplicate using six additional controls (cells in medium). Cell viability was calculated using the following equation: cell viability $(\%)=\left[\mathrm{OD}_{560}\right.$ (sample)/OD 560 (control) $] \times 100$.

2.6. In Vitro Antioxidant Activity. Free radical scavenging activity was evaluated by the 2,2-diphenyl-1-picrylhydrazyl (DPPH) test with modifications [21]. One hundred microliters of RP free, formulations, or control (ethanol; 10$60 \mu \mathrm{g} / \mathrm{mL}$ ) was added to $3.9 \mathrm{~mL}$ of DPPH solution (ethanol; $60 \mu \mathrm{M})$. After 30 minutes storage in a dark place, the absorbance measures were calculated using a spectrophotometer at $517 \mathrm{~nm}$. All measurements were repeated three times. Free radical scavenging activity was calculated using the following formula: \% inhibition DPPH $=\left[\left(A_{0}-A_{1}\right) /\right.$ $\left.A_{0} \times 100\right]$, where $A_{0}$ represents absorbance of control and $A_{1}$ represents absorbance of sample. The $\mathrm{IC}_{50}$ value was determined by plotting concentration of formulations versus the percentage keeping DPPH at a steady state [21].

2.7. Statistical Analyses. Data were analyzed using the mean and standard deviation and compared by analysis of variance (ANOVA). The Tukey test was used to assess significant differences between samples, where values $P<0.05$ were considered statistically significant. The program Origin 7.0 SRO was used for the treatment of the data.

\section{Results and Discussion}

Formulations were prepared with different surfactant/oil/ water ratios, with and without RP. The compositions were based on the pseudoternary phase diagram previously constructed for the same mixture in the experimental conditions [16]. Two formulations were selected for tests-OLS, constituted by $30 \%$ surfactant (polyether functional siloxane, PSF), $25 \%$ of oil phase (silicon glycol copolymer, SGC), and $45 \%$ of water, and TLS-constituted by $40 \%$ PSF, $50 \%$ SGC, and $10 \%$ water. To each of these systems, $1 \%$ RP was added, yielding the formulations $\mathrm{OLS}_{v}$ and $\mathrm{TLS}_{v}$. The development and characterization of the system were crucial to the choice of the formulation study. The ternary phase diagram (Figure 2) obtained had extensive concentration transparent systems (TVS and TLS), since the oil phase of the system also has surfactant properties. At low concentrations of water and oil intermediates OVS and OLS were formed and concentrations of oil and water phase below 30\% were observed PS.

The determination of the optical properties of the formulations was performed observing the sample between crossed polarizers. Lamellar and hexagonal mesophases are anisotropic, while cubic mesophases are isotropic [22, 23]. The presence of Maltese crosses for TLS, Figure 3(a), showed the presence of a lamellar liquid crystal, LCS. The formulation OLS did not present liquid crystalline mesophases, as shown by the homogenous dark field (Figure 3(b)) [18]. 


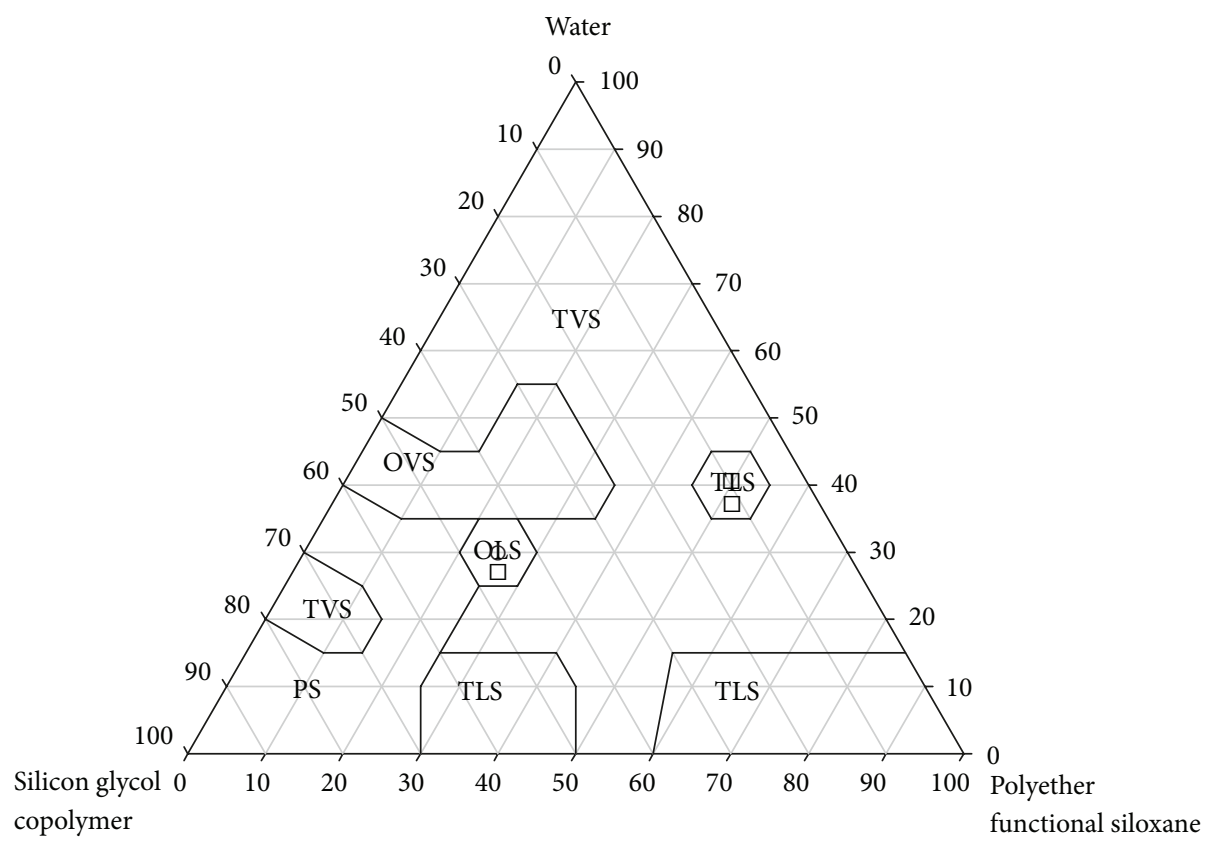

FIGURE 2: Ternary phase diagram, with $O$ and the square $(\square)$ representing the selected regions, where TVS is transparent viscous system, OVS is opaque and viscous system, TLS is transparent liquid system, OLS is opaque liquid system, and PS is phase separation.

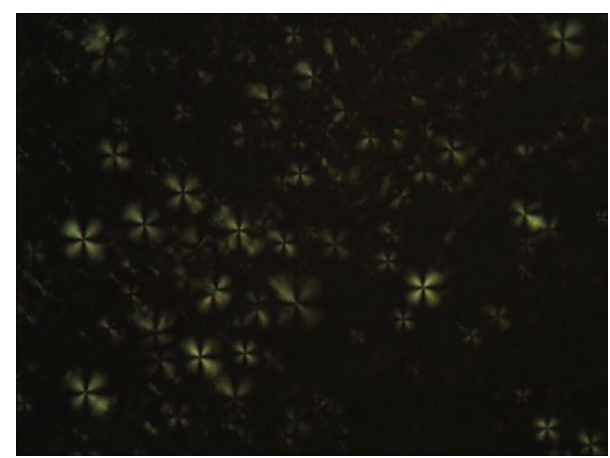

(a)

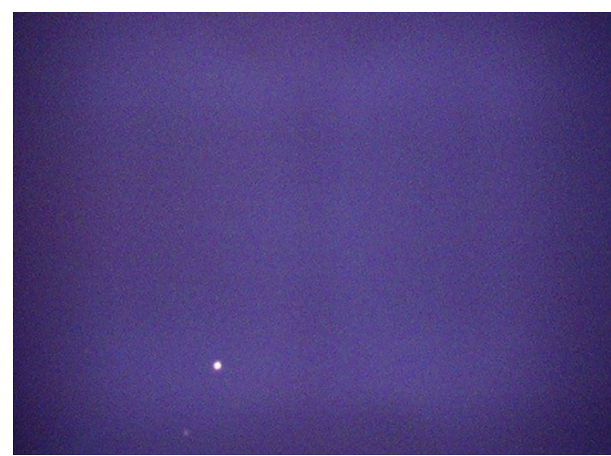

(b)

Figure 3: Images obtained by Polarized light microscopy: (a) transparent liquid system (TLS) showed Maltese crosses; (b) opaque liquid system (OLS) showed dark field. The objects are air bubbles (magnified of 20x).

In the pharmaceutical development of cosmetics, the study of rheology (flow characteristics) has fundamental importance when considering the manufacturing process and the preparation, transportation, storage, and use by consumers $[24,25]$. The rheological analysis was made in the form of rheograms, showing the relationship between shear stress $(\sigma)$ and shear rate $(\gamma)$. In Figure 4 we can observe the relationship between $\sigma$ and $\gamma$ for all of the formulations. For some combinations the values of the shear stress are different for the curves with increased and reduced shear rate. This characteristic is typical of thixotropic materials [26].

The viscosity decreases with increasing shear rate which occurred in the studied system irrespective of the definition of viscosity used and is a characteristic of pseudoplastic fluid [26]. This makes the product suitable for topical use, because of the initial shear to apply the sample; the reduced viscosity results in a good spreading during application and in the formation of uniform film on the skin surface [2729]. In the formulations, by decreasing shear rate the values return to baseline, the rheological behavior is reversible, and the viscosity returns to the initial value sometime after the initial step deformation [30-33]. This phenomenon is also useful for skin applications, since the increased viscosity assists in retaining the formulation in place. As for the OLS sample compared with sample TLS a pronounced decrease in viscosity was observed with increasing shear rate. For both formulations, the addition of vitamin increased the viscosity of the formulations.

The behavior of these formulations can be reaffirmed based on power law, described in the following equation: $\tau=k \cdot \gamma^{\eta}$, where $k$ and $\eta$ values are related to the consistency and flow index, respectively. Thus, in this model, values of $n$ 


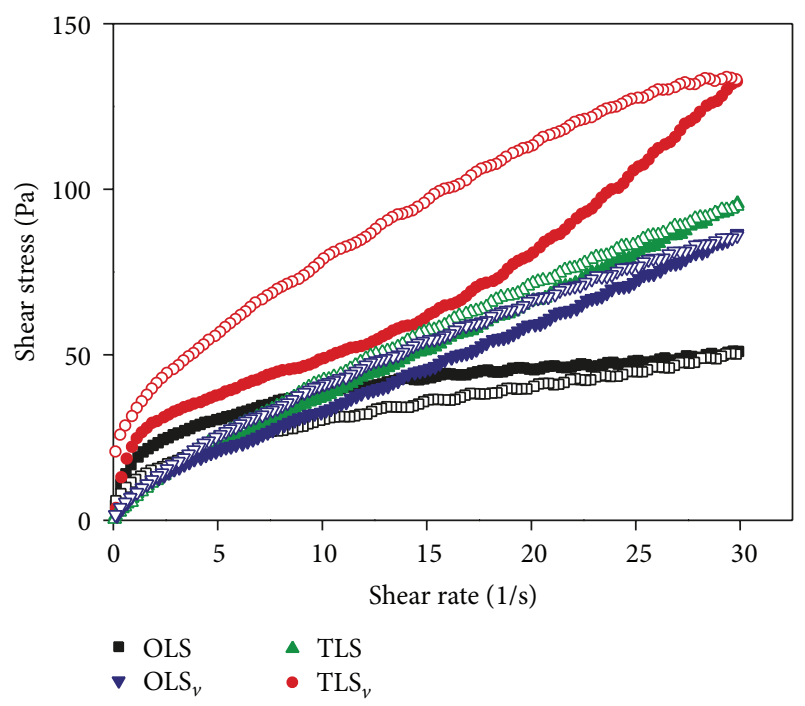

FIGURE 4: Rheogram of the systems studied, where OLS is opaque liquid system, $\mathrm{OLS}_{v}$ is RP-loaded opaque liquid system, TLS is transparent liquid system, and $\mathrm{TLS}_{v}$ is RP-loaded transparent liquid system. The solid symbols represent the upper curves and the hollow symbols represent the lower curves.

TABLE 1: Index of flow $(\eta)$ and consistency index $(k)$ of the formulations.

\begin{tabular}{lcc}
\hline Formulations & $\eta$ & $k$ \\
\hline OLS & 0.28834 & 19.2766 \\
OLSv & 0.82859 & 5.00762 \\
TLS & 0.82742 & 5.60389 \\
TLSv & 0.78206 & 8.36384 \\
\hline
\end{tabular}

OLS: opaque liquid system, OLSv: RP-loaded opaque liquid system, TLS: transparent liquid systems, and TLSv: retinyl palmitate-loaded transparent liquid systems.

greater than 1 represent dilatant fluid, values of $n$ smaller than 1 represent a pseudoplastic fluid, and finally values of $n$ equal to 1 represent Newtonian fluid [34].

In Table 1 , the values of $\eta$ and $k$ for all formulations as compared to the rating of the rheogram are presented.

The formulations outlined in Table 1 (OLS, OLS $v$, TLS, and $\mathrm{TLS}_{v}$ ) show that all values of $\eta$ are smaller than 1 , indicating pseudoplastic fluids which agree with the interpretation based on the observation of the rheogram of Figure 4. In OLS, after adding RP, there was an increase in the value of $\eta$, from 0.28834 to 0.82859 . Nevertheless, the same was not observed with TLS and TLS ${ }_{v}$, and close values were found among them.

3.1. In Vitro Biological Assays. The in vitro test is important for screening a substance which can be used subsequently in preclinical trials. In addition, it is able to provide initial parameters such as viability and therapeutic targets for subsequent reviews [35].

The materials used in the formulations present wellknown safety profiles; however, these mixtures have been shown to form structures which may change the barrier

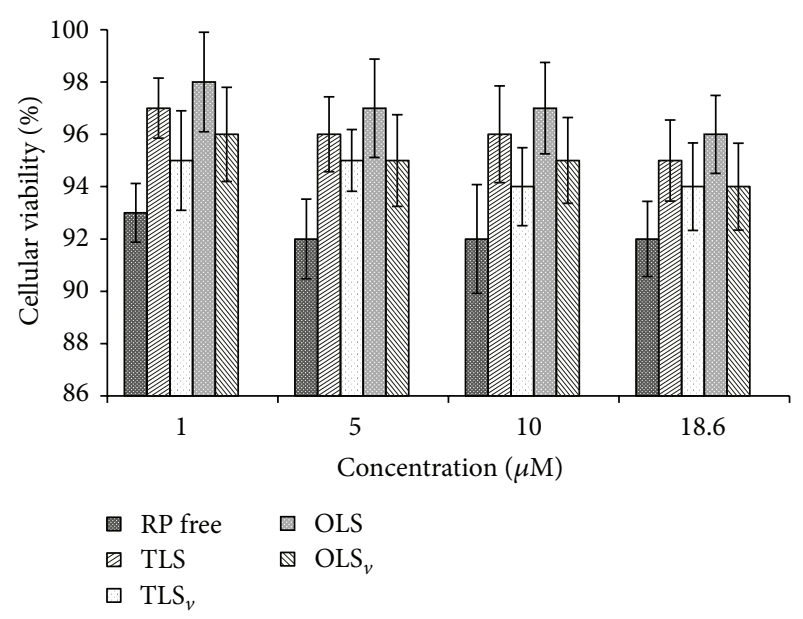

FIGURE 5: Percentage of cell viability of RP free, transparent liquid system (TLS), opaque liquid system (OLS), RP-loaded transparent liquid system $\left(\mathrm{TLS}_{v}\right)$, and RP-loaded opaque liquid system $\left(\mathrm{OLS}_{v}\right)$.

properties of the skin. Therefore, it is important to study the safety of these new systems on the skin.

Erythrocyte membranes have been broadly used for in vitro cytotoxicity assays because they are easily isolated by centrifugation and obtained by venipuncture. They represent a good model to evaluate the interaction of drugs with the membrane and thus provide information about changes in the composition of lipids, enzymes, or other membrane proteins [36].

The safety profile was assessed using the hemolysis of red blood cells and in vitro cytotoxicity assays. Free RP caused $4.69 \pm 0.54 \%$ lysis of erythrocyte membranes. TLS and OLS caused lysis of $1.23 \% \pm 0.69$ and $1.49 \pm 0.35 \%$ of the erythrocyte membranes, respectively. The incorporation

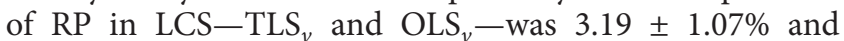
$3.42 \pm 0.54 \%$, respectively and showed decreased erythrocyte lysis compared to the free RP. Thus, all systems showed a tolerable hemolysis of erythrocytes. The positive control was represented by $100 \%$ hemolysis of erythrocytes using Triton X-100, a known hemolytic agent, thus validating the experiment. The study results indicate that the treatment developed with lipid systems showed less toxicity, which is a potential alternative to therapeutic applications [20].

Similarly, the safety profile of praziquantel (PZQ) was evaluated in studies using red cell hemolysis and showed that the encapsulation of PZQ in nanostructured lipid carriers improved the safety profile of the drug with decreased lysis of erythrocytes in relation to the free PZQ. The systems demonstrated improved efficacy in comparison with free PZQ [37].

In vitro cytotoxicity was performed using J-774 mouse macrophages as a cellular model. The data are shown as a percent of cellular viability (Figure 5).

Abbasalipourkabir et al. (2011) say that some factors may influence the cytotoxic effect of the particles: adherence to the cell membrane, particle internalization, and degradation products in the cell culture medium or in the intracellular 


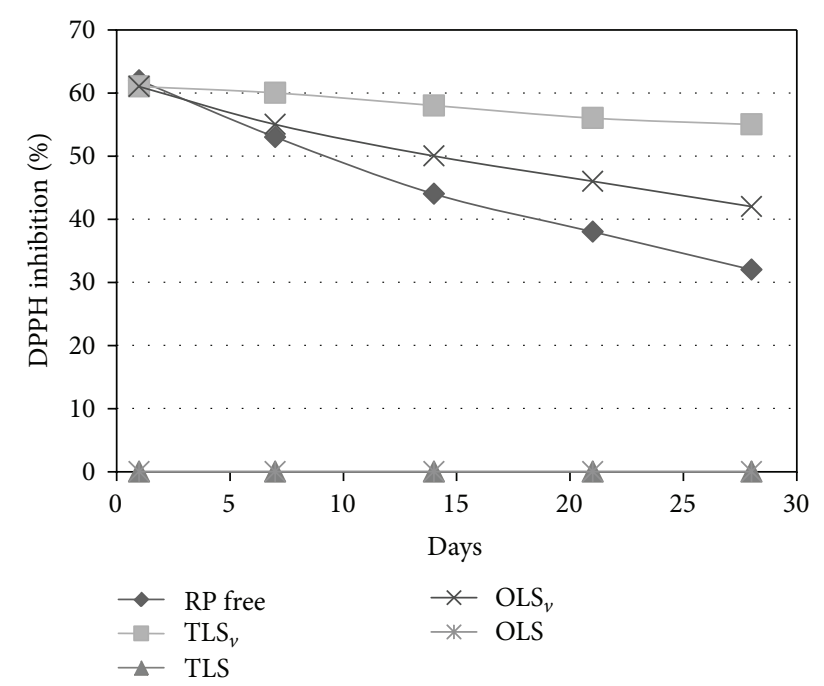

FIGURE 6: Percentage of inhibition of 2,2-diphenyl-1-picrylhydrazyl (DPPH) radical by formulations over a period of 28 days. RP free: retinyl palmitate free, $\mathrm{TLS}_{v}$ : retinyl palmitate-loaded transparent liq-

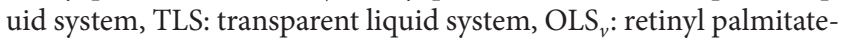
loaded opaque liquid system, and OLS: opaque liquid system.

environment [38]. Nevertheless, cell types may exhibit different changes in susceptibility to particulate carriers since the system contains natural lipids to be well tolerated by the body [39].

Cell viability showed that free RP did not kill normal macrophages presenting a result superior to $92 \%$. Moreover, the RP-unloaded and RP-loaded CLS also did not show toxic activity. Therefore, the results indicate the safety and biocompatibility of the formulations and free RP for eukaryotic cells [37].

The formulations with or without the addition of RP were evaluated for in vitro antioxidant activity over a period of 28 days. The results obtained are shown in Figure 6.

The antioxidant activity was measured based on the methodology of Blois [21], who uses the stable radical DPPH which is reduced by antioxidants. The results were expressed as DPPH inhibition (\%).

It can be seen that over the period of 28 days there was a decrease in antioxidant power for all the experimental groups, with the most marked decrease for the RP free and a statistical difference between the samples $(P<0.05)$. The lesser destabilization of the samples is probably due to increased stabilization of the vitamin in the formulations. Moreover, the antioxidant activity was greater for $\mathrm{TLS}_{v}$ compared to $\mathrm{OLS}_{v}$, suggesting increased stability of the vitamin in the lamellar LCS. This effect has been found in the literature; the liquid crystals can protect the formulation while preventing the degradation of the active ingredient $[13,16,35,40,41]$.

Therefore, all formulations maintained the antioxidant properties of the RP, which makes them promising as vehicles for incorporation of the vitamin, aiming its topical application at the treatment of aging skin.

\section{Conclusion}

The results suggest that the LCS containing polyether functional siloxane (PFS) as surfactant, silicon glycol copolymer (SGC) as oil phase, and water in the ratios $40: 50: 10$ with RP $\left(\mathrm{TLS}_{v}\right.$ ) presented a lamellar phase and pseudoplastic behavior in rheological analysis. In vitro safety profile showed that this formulation is not cytotoxic and in vitro antioxidant activity suggested that LCS presented a higher capacity to maintain the antioxidant activity of RP. PFS-based systems may be a promising platform for RP topical application for the treatment of skin aging.

\section{Conflict of Interests}

The authors declare that there is no conflict of interests regarding the publication of this paper.

\section{Acknowledgments}

This work was financially supported by São Paulo Research Foundation (FAPESP), Conselho Nacional de Desenvolvimento Científico e Tecnológico (CNPq) and Programa de Apoio ao Desenvolvimento Científico (PADC-UNESP).

\section{References}

[1] Y. R. Helfrich, D. L. Sachs, and J. J. Voorhees, "Overview of skin aging and photoaging," Dermatology Nursing, vol. 20, no. 3, pp. 177-184, 2008.

[2] M. Manela-Azulay and E. Bagatin, "Cosmeceuticals vitamins," Clinics in Dermatology, vol. 27, no. 5, pp. 469-474, 2009.

[3] O. Sorg, S. Kuenzli, G. Kaya, and J. H. Saurat, "Proposed mechanisms of action for retinoid derivatives in the treatment of skin aging," Journal of Cosmetic Dermatology, vol. 4, no. 4, pp. 237-244, 2005.

[4] M. Ramos-e-Silva, D. M. Hexsel, M. S. Rutowitsch, and M. Zechmeister, "Hydroxy acids and retinoids in cosmetics," Clinics in Dermatology, vol. 19, no. 4, pp. 460-466, 2001.

[5] L. Rittié and G. J. Fisher, "UV-light-induced signal cascades and skin aging," Ageing Research Reviews, vol. 1, no. 4, pp. 705-720, 2002.

[6] M. P. Lupo, "Antioxidants and vitamins in cosmetics," Clinics in Dermatology, vol. 19, no. 4, pp. 467-473, 2001.

[7] R. Serri and M. Iorizzo, "Cosmeceuticals: focus on topical retinoids in photoaging," Clinics in Dermatology, vol. 26, no. 6, pp. 633-635, 2008.

[8] M.-S. Lee, K.-H. Lee, H.-S. Sin, S.-J. Um, J.-W. Kim, and B.K. Koh, "A newly synthesized photostable retinol derivative (retinyl N-formyl aspartamate) for photodamaged skin: profilometric evaluation of 24-week study," Journal of the American Academy of Dermatology, vol. 55, no. 2, pp. 220-224, 2006.

[9] J. Zussman, J. Ahdout, and J. Kim, "Vitamins and photoaging: do scientific data support their use?" Journal of the American Academy of Dermatology, vol. 63, no. 3, pp. 507-525, 2010.

[10] D. A. Glaser, "Anti-aging products and cosmeceuticals," Facial Plastic Surgery Clinics of North America, vol. 12, no. 3, pp. 363$372,2004$.

[11] C. Antille, C. Tran, O. Sorg, P. Carraux, L. Didierjean, and J.H. Saurat, "Vitamin A exerts a photoprotective action in skin 
by absorbing ultraviolet B radiation," Journal of Investigative Dermatology, vol. 121, no. 5, pp. 1163-1167, 2003.

[12] G. G. Morais, O. D. H. Santos, W. P. Oliveira, and P. A. Rocha Filho, "Attainment of $\mathrm{O} / \mathrm{W}$ emulsions containing liquid crystal from annatto oil (Bixa orellana), coffee oil, and tea tree oil (Melaleuca alternifolia) as oily phase using HLB system and ternary phase diagram," Journal of Dispersion Science and Technology, vol. 29, no. 2, pp. 297-306, 2008.

[13] B. V. Bonifácio, P. B. da Silva, M. A. Dos et al., "Nanotechnologybased drug delivery systems and herbal medicines: a review," International Journal of Nanomedicine, pp. 1-15, 20149.

[14] M. Kreilgaard, "Influence of microemulsions on cutaneous drug delivery," Advanced Drug Delivery Reviews, vol. 54, pp. S77-S98, 2002.

[15] D. I. Nesseem, "Formulation and evaluation of itraconazole via liquid crystal for topical delivery system," Journal of Pharmaceutical and Biomedical Analysis, vol. 26, no. 3, pp. 387-399, 2001.

[16] P. S. Prestes, M. Chorilli, L. A. Chiavacci, M. V. Scarpa, and G. R. Leonardi, "Physicochemical characterization and rheological behavior evaluation of the liquid crystalline mesophases developed with different silicones," Journal of Dispersion Science and Technology, vol. 31, no. 1, pp. 117-123, 2009.

[17] M. Chorilli, P. S. Prestes, R. B. Rigon et al., "Structural characterization and in vivo evaluation of retinyl palmitate in non-ionic lamellar liquid crystalline system," Colloids and Surfaces B, vol. 85, no. 2, pp. 182-188, 2011.

[18] M. Chorilli, P. S. Prestes, R. B. Rigon, G. R. Leonardi, L. A. Chiavacci, and M. V. Scarpa, "Desenvolvimento de sistemas líquido-cristalinos empregando silicone fluido de co-polímero glicol e poliéter funcional siloxano," Química Nova, vol. 32, no. 4, pp. 1036-1040, 2009.

[19] M. Jumaa, P. Kleinebudde, and B. W. Müller, "Physicochemical properties and hemolytic effect of different lipid emulsion formulations using a mixture of emulsifiers," Pharmaceutica Acta Helvetiae, vol. 73, no. 6, pp. 293-301, 1999.

[20] Z.-R. Huang, S.-C. Hua, Y.-L. Yang, and J.-Y. Fang, "Development and evaluation of lipid nanoparticles for camptothecin delivery: a comparison of solid lipid nanoparticles, nanostructured lipid carriers, and lipid emulsion," Acta Pharmacologica Sinica, vol. 29, no. 9, pp. 1094-1102, 2008.

[21] M. S. Blois, "Antioxidant determinations by the use of a stable free radical," Nature, vol. 181, no. 4617, pp. 1199-1200, 1958.

[22] T. Norling, P. Lading, S. Engstrom, K. Larsson, N. Krog, and S. S. Nissen, "Formulation of a drug delivery system based on a mixture of monoglycerides and triglycerides for use in the treatment of periodontal disease," Journal of Clinical Periodontology, vol. 19, no. 9, pp. 687-692, 1992.

[23] T. P. Formariz, M. C. C. Urban, A. A. da Silva Júnior, M. P. D. Gremião, and A. G. de Oliveira, "Microemulsões e fases líquidas cristalinas como sistemas de liberação de fármacos," Revista Brasileira de Ciências Farmacêuticas, vol. 41, pp. 301-313, 2005.

[24] I. M. Number, 3: An Introduction to Rheology, Micelle Press, Dorset, UK, 1997.

[25] G. Schramm, Reologia e Reometria: Fundamentos Teóricos e Práticos, Artliber, 2006.

[26] A. L. K. Milan, D. Milão, A. A. Souto, and T. W. F. Corte, "Estudo da hidratação da pele por emulsões cosméticas para xerose e sua estabilidade por reologia," Brazilian Journal of Pharmaceutical Sciences, vol. 43, no. 4, 2007.

[27] M. H. Lahoud and R. Campos, "Aspectos teóricos relacionados à reologia farmacêutica," Visão Acadêmica, vol. 11, no. 1, 2011.
[28] M. Chorilli, R. B. Rigon, G. Calixto et al., "Rheological characterization and safety evaluationof non-ionic lamellar liquid crystalline systems containing retinyl palmitate," Journal of Biomedical Nanotechnology. In press.

[29] L. R. Gaspar and P. M. B. G. Maia Campos, "Rheological behavior and the SPF of sunscreens," International Journal of Pharmaceutics, vol. 250, no. 1, pp. 35-44, 2003.

[30] T. Gao, J. Tien, and Y. Choi, "Sunscreen formulas with multilayer lamella structure," Cosmetics and Toiletries, vol. 118, no. 10, pp. 41-52, 2003.

[31] F. C. Carvalho, G. Calixto, I. N. Hatakeyama, G. M. Luz, M. P. Gremião, and M. Chorilli, "Rheological, mechanical, and bioadhesive behavior of hydrogels to optimize skin delivery systems," Drug Development and Industrial Pharmacy, vol. 39, no. 11, pp. 1750-1757, 2013.

[32] I. Almeida and M. F. Bahia, "Reologia: interesse e aplicações na área cosmético-farmacêutica," Cosmetics \& Toiletries, vol. 15, no. 3, pp. 96-100, 2003.

[33] P. S. Prestes, R. B. Rigon, G. N. Guimarães et al., "Development, physical-chemical stability and rheological behavior of silicones formulations containing Dimethylaminoethanol (DMAE)," Journal of Applied Pharmaceutical Science, vol. 3, no. 2, pp. 1-5, 2013.

[34] F. C. Carvalho, Desenvolvimento e caracterização de sistemas nanoestruturados para potencial administração nasal de zidovudina [Teses e Dissertações], Universidade Estadual Paulista, 2009.

[35] M. Gonçalez, M. Corrêa, and M. Chorilli, "Skin delivery of kojic Acid-loaded nanotechnology-based drug delivery systems for the treatment of skin aging," BioMed Research International, vol. 2013, Article ID 271276, 9 pages, 2013.

[36] S. V. P. Malheiros, N. C. Meirelles, and E. de Paula, "Pathways involved in trifluoperazine-, dibucaine- and praziquantelinduced hemolysis," Biophysical Chemistry, vol. 83, no. 2, pp. 89$100,2000$.

[37] F. Kolenyak-Santos, C. Garnero, R. N. de Oliveira et al., "Nanostructured lipid carriers as a strategy to improve the in vitro schistosomiasis activity of praziquantel," Journal of Nanoscience and Nanotechnology, vol. 14, pp. 1-12, 2014.

[38] R. Abbasalipourkabir, A. Salehzadeh, and R. Abdullah, "Cytotoxicity effect of solid lipid nanoparticles on human breast cancer cell lines," Biotechnology, vol. 10, no. 6, pp. 528-533, 2011.

[39] A. C. Silva, D. Santos, D. C. Ferreira, and E. B. Souto, "Minoxidil-loaded nanostructured lipid carriers (NLC): characterization and rheological behaviour of topical formulations," Die Pharmazie, vol. 64, no. 3, pp. 177-182, 2009.

[40] O. D. H. dos Santos, M. F. P. de Camargo, F. F. de Andrade, and P. A. da Rocha Filho, "Study of liquid-crystalline phase changes during evaporation in vegetable oil emulsions," Journal of Dispersion Science and Technology, vol. 27, no. 7, pp. 997-1001, 2006.

[41] M. H. Oyafuso, F. C. Carvalho, L. A. Chiavacci, M. P. D. Gremião, and M. Chorilli, "Design and characterization of silicone and surfactant based systems for topical drug delivery," Journal of Nanoscience and Nanotechnology, vol. 14, pp. 1-10, 2014. 

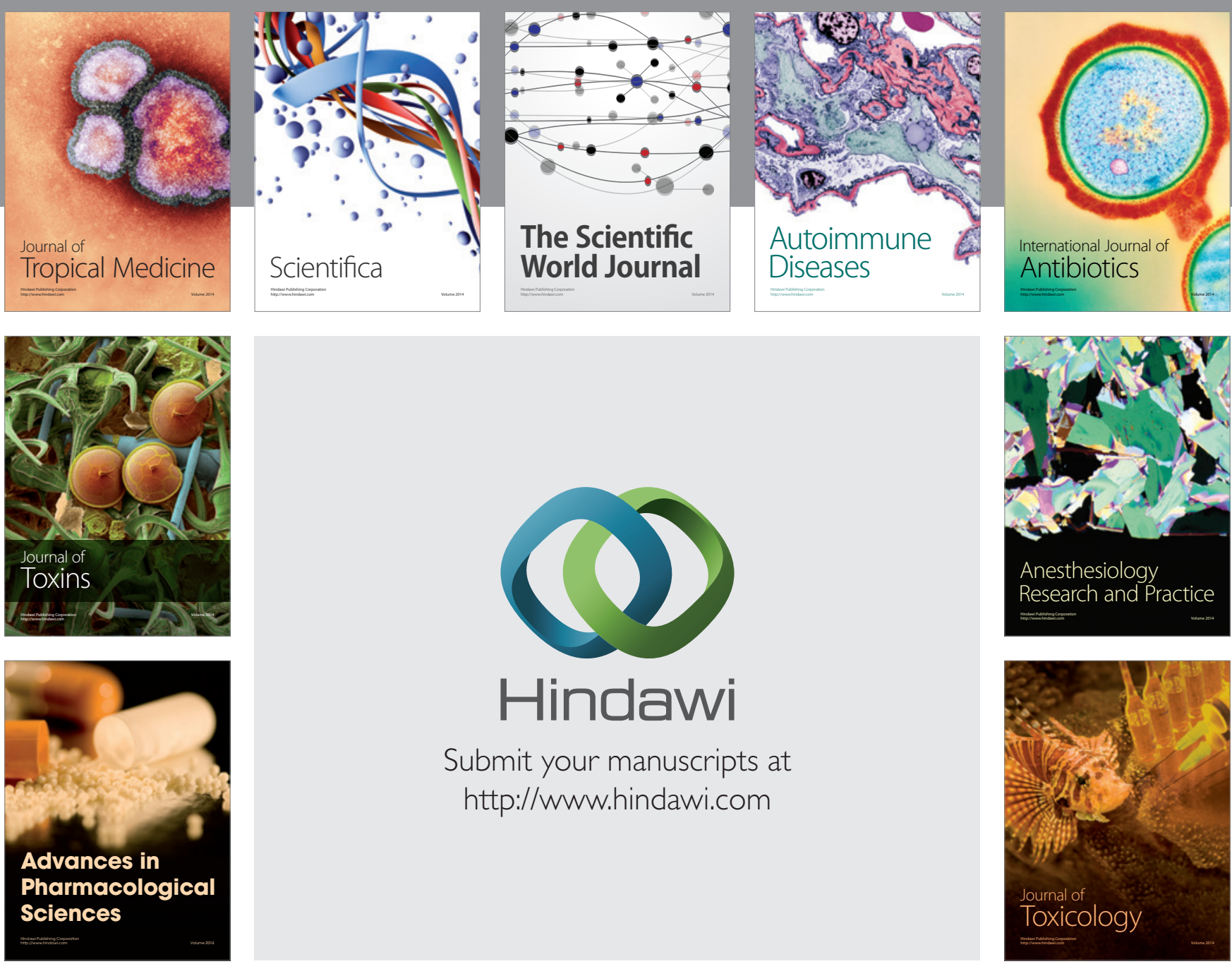

\section{Hindawi}

Submit your manuscripts at

http://www.hindawi.com
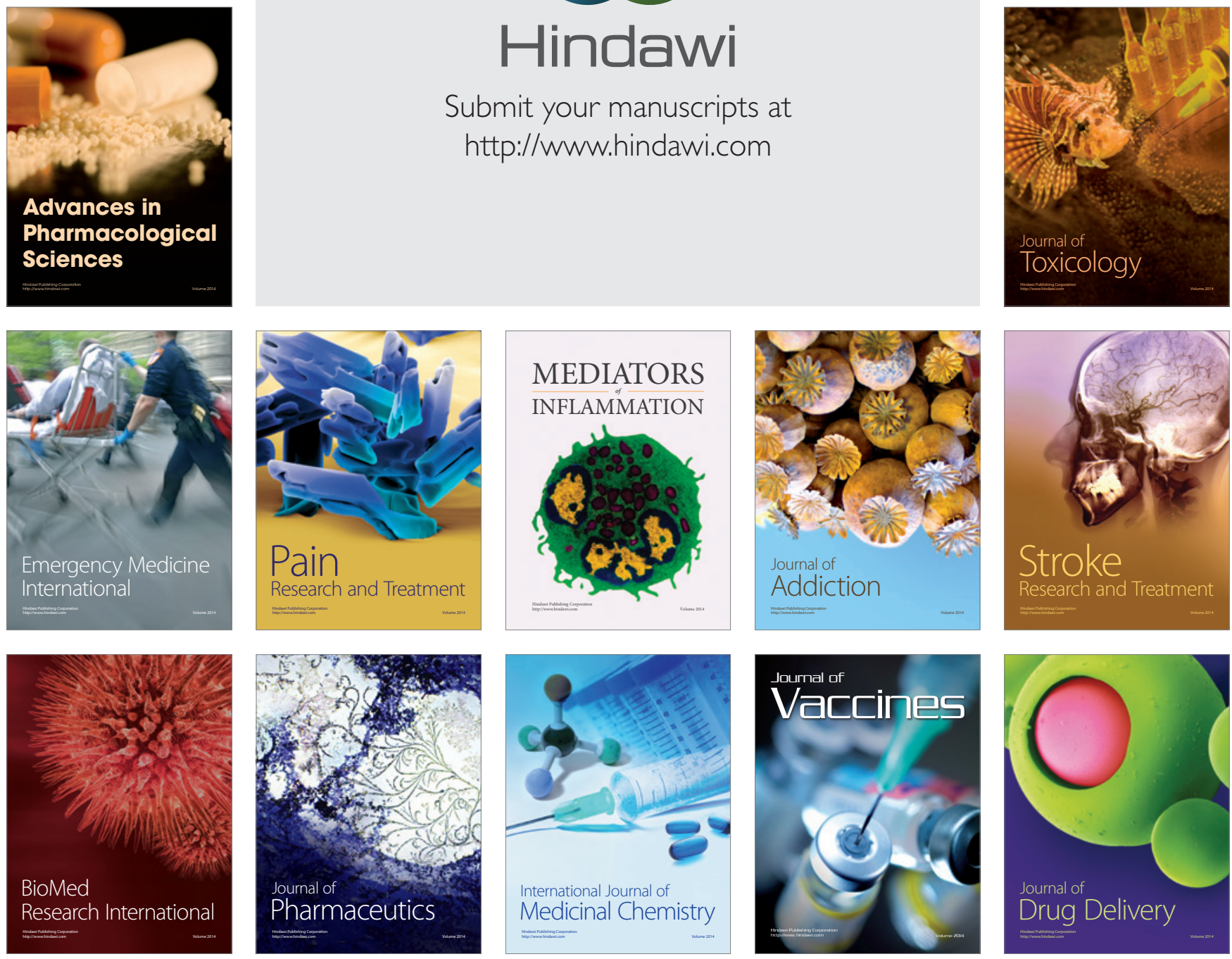